Pacific Journal of Mathematics

ON THE DIVISORS OF MONIC POLYNOMIALS OVER A 


\section{ON THE DIVISORS OF MONIC POLYNOMIALS OVER A COMMUTATIVE RING}

\section{Robert Gilmer aNd William Heinzer}

For a commutative ring $R$ with identity, denote by $R\langle X\rangle$ the quotient ring of $R[X]$ with respect to the regular multiplicative system $S$ of monic polynomials over $R$. The present paper determines the group of units of the ring $R\langle X\rangle$. This is equivalent to the problem of determining the saturation $S^{*}$ of the multiplicative system $S$; by definition, $S^{*}$ consists of all divisors of monic polynomials over $R$. For a nonzero polynomial

$$
f=f_{0}+f_{1} X+\cdots+f_{n} X^{n} \in R[X],
$$

it is shown that each of the following conditions (A) and $(B)$ is equivalent to the condition that $f$ divides a monic polynomial over $R$ (in (B), the ring $R$ is reduced).

(A) The coefficients of $f$ generate the unit ideal of $R$ and, for each $j$ between 0 and $n$ and for each prime ideal $P$ of $R$, the relations $f_{j+1}, \cdots, f_{n} \in P, f_{j} \notin P$, imply that $f_{j}$ is a unit modulo $P$.

(B) There exists a direct sum decomposition

$$
R=R_{1} \oplus \cdots \oplus R_{m} \text { of } R
$$

such that if $f=g_{1}+\cdots+g_{m}$ is the decomposition of $f$ with respect to the induced decomposition

$$
R[X]=R_{1}[X] \oplus \cdots \oplus R_{m}[X] \text { of } R[X],
$$

then the leading coefficient of $g_{i}$ is a unit of $R_{i}$ for each $i$.

One corollary to the preceding characterizations is that $S^{*}$ is the set of polynomials over $R$ with unit leading coefficient if and only if the ring $R$ is reduced and indecomposable.

Let $R$ be a commutative ring with identity, let $X$ be an indeterminate over $R$, and denote by $S$ the regular multiplicative system of monic polynomials over $R$. The quotient ring $R[X]_{S}$ of $R[X]$ is currently receiving attention, probably because of the role it plays in Quillen's solution of the Serre Conjecture [6]. We use the symbol $R\langle X\rangle$ to denote the ring $R[X]_{s}$; this differs from Quillen's notation $R(X)$ for this ring, but our choice of notation is based on the fact that $R(X)$ has traditionally been used to denote the quotient ring of $R[X]$ with respect to the multiplicative system of polynomials of unit content [5, p. 18], [2, §33]. The aim of this paper is to determine the group of units of the ring $R\langle X\rangle$. If $S^{*}=$ $\{f \in R[X] \mid f$ divides an element of $S\}$ (that is, $S^{*}$ is the saturation 
of $S$ ), then it is well known that the group of units of $R\langle X\rangle$ is $\left\{f / g \mid f, g \in S^{*}\right\}$, the quotient group of the cancellative abelian semigroup $S^{*}$. Hence, the problem of determining the units of $R\langle X\rangle$ is equivalent to the problem of determining the set of divisors of monic polynomials of $R[X]$, and we approach the problem from this perspective. An obvious family of divisors of monic polynomials is the family of polynomials with unit leading coefficient; to wit, if $f=f_{0}+f_{1} X+\cdots+f_{n} X^{n}$, where $f_{n}$ is a unit of $R$, then $f$ divides the monic polynomial $f_{n}^{-1} f$. We denote by $\mathscr{S}(R[X])$ the set of polynomials with unit leading coefficient. The set $\mathscr{P}(R[X])$ is again a regular multiplicative system in $R[X]$, and $S$ and $\mathscr{S}(R[X])$ have the same saturation. We are therefore concerned with the problem of determining the saturation of $\mathscr{S}(R[X])$. There are some cases (such as that in which $R$ is an integral domain) where it is clear that $\mathscr{S}(R[X])$ itself is saturated; in Corollary 7 we prove that $\mathscr{P}(R[X])$ is saturated if and only if the ring $R$ is indecomposable and reduced. In the general case we prove in Theorem 6 that a polynomial $f=f_{0}+f_{1} X+\cdots+f_{m} X^{m}$ belongs to the saturation of $\mathscr{S}(R[X])$ if and only if (1) the coefficients of $f$ generate the unit ideal of $R$, and (2) for each $j$ between 0 and $n$ and for each prime ideal $P$ of $R$, the relations $f_{j+1}, \cdots, f_{n} \in P, f_{j} \notin P$ imply that $f_{j}$ is a unit modulo $P$. An alternate description of the saturation of $\mathscr{S}(R[X])$ is provided in Theorem 9 in the case where $R$ is reduced.

Before proceeding further, we introduce some simplifying terminology and notation. If $g \in R[X]$, then the ideal of $R$ generated by the coefficients of $g$ is called the content of $g$ and is denoted by $C(g)$; the polynomial $g$ has unit content if $C(g)=R$. The set $U$ of polynomials $g \in R[X]$ of unit content forms a saturated multiplicative system in $R[X]$, and the quotient ring $R[X]_{U}$ is traditionally denoted by $R(X)[2,(33.1)]$. Since $\mathscr{S}(R[X]) \subseteq U$, the saturation of $\mathscr{S}(R[X])$ is also contained in $U$. If $A$ is a proper ideal of $R$, then we denote by $\phi_{A}$ the canonical homomorphism of $R$ onto $R / A$ and by $\phi_{A}^{*}$ the unique extension of $\phi_{A}$ to a homomorphism of $R[X]$ onto $(R / A)[X]$ mapping $X$ to $X$. It is clear that $\phi_{A}^{*}$ maps $\mathscr{S}(R[X])$ into $\mathscr{S}((R / A)[X])$ for each proper ideal $A$ of $R$. The main result we seek is Theorem 6; the observations of this paragraph imply that each element of the saturation of $\mathscr{S}(R[X])$ satisfies conditions (1) and (2) of the preceding paragraph.

Proposition 1. Assume that $f=f_{0}+f_{1} X+\cdots+f_{n} X^{n}$ belongs to the saturation of $\mathscr{S}(R[X])$. Then (1) $f$ has unit content, and (2) for each $j$ between 0 and $n$ and for each prime ideal $P$ of $R$, the relations $f_{j+1}, \cdots, f_{n} \in P, f_{j} \notin P$ imply that $f_{j}$ is a unit modulo $P$. 
Proof. We have already observed that the saturation of $\mathscr{S}(R[X])$ is contained in the multiplicative system of polynomials with unit content - that is, that (1) is satisfied. In relation to (2), we remark that for $j=n$, the condition is to be interpreted as meaning that $f_{n}$ is a unit modulo $P$ for each prime $P$ of $R$ such that $f_{n} \notin P$. Assume that $P$ is prime and that $f_{j+1}, \cdots, f_{n} \in P$, while $f_{j} \notin P$. Then $\phi_{P}^{*}(f)$ is an element of $\mathscr{S}((R / P)[X])$ with leading coefficient $\phi_{P}\left(f_{j}\right)$. Since $R / P$ is an integral domain, the multiplicative system $\mathscr{S}((R / P)[X])$ is saturated. Therefore $\phi_{P}\left(f_{j}\right)$ is a unit of $R / P$; that is, $f_{j}$ is a unit modulo $P$.

For the sake of brevity, we call a polynomial satisfying (1) and (2) of Proposition 1 a (*)-polynomial. The next result shows that $f$ and $\phi_{N}^{*}(f)$, where $N$ is the nilradical of $R$, are simultaneously $(*)$ polynomials.

Proposition 2. Let $N$ be the nilradical of the ring $R$, and let $\dot{\rho}_{*}^{*}$ be the canonical homomorphism of $R[X]$ onto $(R / N)[X] . \quad$ If $f \in R[X]$, then $f$ is a (*)-polynomial if and only if $\phi_{N}^{*}(f)$ is a (*)polynomial.

Proof. Let $f=f_{0}+f_{1} X+\cdots+f_{n} X^{n}$. We prove that $f$ is a (*)-polynomial if

$$
\phi_{N}^{*}(f)=\dot{\phi}_{N}\left(f_{0}\right)+\phi_{N}\left(f_{1}\right) X+\cdots+\dot{\phi}_{N}\left(f_{n}\right) X^{n}
$$

is a $(*)$-polynomial; the proof of the converse is similar and will be omitted. Thus, $\left(f_{0}, \cdots, f_{n}\right)+N=R$ since $\phi_{N}^{*}(f)$ has unit content. Therefore no maximal ideal of $R$ contains $\left(f_{0}, \cdots, f_{n}\right)$ and $N$. Since each maximal ideal of $R$ contains $N$, if follows that no maximal ideal of $R$ contains $\left(f_{0}, f_{1}, \cdots, f_{n}\right)$. That is, $\left(f_{0}, f_{1}, \cdots, f_{n}\right)=R$ and $f$ has unit content. Assume that $j$ is between 0 and $n$ and that $P$ is a prime ideal of $R$ such that $f_{j+1}, \cdots, f_{n} \in P$, while $f_{j} \notin P$. Then $P / N$ is a prime ideal of $R / N$ and the relations

$$
\phi_{N}\left(f_{j+1}\right), \cdots, \phi_{N}\left(f_{n}\right) \in P / N, \quad \phi_{N}\left(f_{j}\right) \notin P / N
$$

are satisfied. This means, by assumption, that $\dot{\phi}_{N}\left(f_{j}\right)$ is a unit modulo $P / N$, and hence $f_{j}$ is a unit modulo $P$. Therefore $f$ is a (*)-polynomial, as was to be proved.

In order to prove that the saturation of $\mathscr{P}(R[X])$ consists of the set of (*)-polynomials of $R[X]$, Proposition 2 and the next result will show that it is sufficient to consider the case where $R$ is a reduced ring.

Proposition 3. Let $N$ be the nilradical of $R$, and let $\phi_{v}^{*}$ denote 
the canonical homomorphism of $R[X]$ onto $(R / N)[X]$. If $f \in R[X]$, then $f$ belongs to the saturation of $\mathscr{S}(R[X])$ if and only if $\phi_{N}^{*}(f)$ belongs to the saturation of $\mathscr{S}((R / N)[X])$.

Proof. If $f$ divides the monic polynomial $g \in R[X]$, then $\phi_{N}^{*}(g) \in$ $(R / N)[X]$ is monic and $\phi_{N}^{*}(f)$ divides $\phi_{N}^{*}(g)$. Hence $\phi_{N}^{*}(f) \in \mathscr{S}((R / N)[X])$. Conversely, if $\phi_{N}^{*}(f)$ belongs to the saturation of $\mathscr{S}\left(\left(R_{/} / N\right)[X]\right)$, then it follows that there exists a monic polynomial $g \in R[X]$ and a polynomial $h \in R[X]$ such that $g-f h \in N[X]$, the kernel of $\phi_{N}^{*}$. Therefore $g-f h$ is nilpotent. If $(g-f h)^{k}=0$, then we have $0 \equiv$ $(g-f h)^{k} \equiv g^{k}$ modulo $(f)$; that is, $f$ divides the monic polynomial $g^{k}$, and $f$ is in the saturation of $\mathscr{S}(R[X])$. This completes the proof of Proposition 3.

For a reduced ring $R$, the leading coefficient of each (*)-polynomial over $R$ generates an idempotent ideal of $R$; this follows from Proposition 1 and the next result, Proposition 4 .

Proposition 4. Assume that $R$ is a reduced ring and that the element $b$ of $R$ is such that $b$ is a unit modulo each prime ideal $P$ of $R$ such that $b \notin P$. Then $b$ generates an idempotent ideal of $R$.

Proof. It suffices to prove that the ideal $(b)$ is locally idempotent. Thus, let $M$ be a maximal ideal of $R$ and let $\mu$ be the canonical homomorphism of $R$ into $R_{M}$. If $b \notin M$, then $(\mu(b))=R_{M}=$ $\left(\mu\left(b^{2}\right)\right)$. If $b \in M$, then $b$ is not a unit modulo any prime ideal contained in $M$, and hence the hypothesis implies that $b$ belongs to each prime of $R$ contained in $M$. Consequently, $\mu(b)$ belongs to the nilradical of $R_{M}$, a reduced ring. Therefore $(\mu(b))=(0)=\left(\mu\left(b^{2}\right)\right)$, and this completes the proof that $(b)$ is locally idempotent.

We remark that the converse of Proposition 4 also holds: if (b) is idempotent, then $b$ is a unit modulo each prime ideal of $R$ that does not contain $b$. We use Proposition 4 to obtain a direct sum decomposition of the ring $R$; the next result, Proposition 5, describes the behavior of the saturation of $\mathscr{S}(R[X])$ with respect to the induced decomposition of $R[X]$. The proof of Proposition 5 is standard, and is therefore omitted.

Proposition 5. Assume that $R$ is the direct sum of its finite family $\left\{R_{i}\right\}_{i=1}^{n}$ of ideals. Let $f \in R[X]$, and let $f=f_{1}+\cdots+f_{n}$ be the decomposition of $f$ with respect to the induced decomposition $R[X]=$ $R_{1}[X] \oplus \cdots \oplus R_{n}[X]$ of $R[X]$. Then $f$ belongs to the saturation of $\mathscr{S}(R[X])$ if and only if $f_{i}$ belongs to the saturation of $\mathscr{S}\left(R_{i}[X]\right)$ for each $i$. 
Proposition 5 is the final preliminary result needed for the proof of Theorem 6.

THEOREM 6. The saturation of $\mathscr{S}(R[X])$ is the set of (*)-polynomials over $R$.

Proof. Proposition 1 shows that each element of the saturation of $\mathscr{S}(R[X])$ is a (*)-polynomial. Conversely, assume that $f=f_{0}+$ $f_{1} X+\cdots+f_{n} X^{n}$ is a (*)-polynomial. To prove that $f$ is in the saturation of $\mathscr{S}(R[X])$, if suffices to prove that $\phi_{N}^{*}(f)($ a $(*)$-polynomial by Proposition 2$)$ is in the saturation of $\mathscr{S}((R / N)[X])$, where $N$ is the nilradical of $R$. Therefore we assume without loss of generality that $R$ is reduced; the proof that $f$ is in the saturation of $\mathscr{S}(R[X])$ is by induction of $n$. For $n=0$, it follows that $f=f_{0}$ is a unit of $R$ since $f$ has unit content. Therefore, $f$ is in $\mathscr{S}(R[X])$. We assume that $(*)$-polynomials of degree less than $n$ are in the saturation of $\mathscr{S}(R[X])$. Then for $f$ of degree $n$, Proposition 4 implies that the ideal $\left(f_{n}\right)$ is idempotent. If $f_{n}$ is a unit of $R$, then $f \in \mathscr{S}(R[X])$ and the proof is complete. Otherwise, $A=\left(f_{n}\right)$ is a proper ideal of $R$ and $R=A \oplus B$, where $B=\operatorname{Ann}\left(f_{n}\right)$. For each $i$ between 0 and $n$, write $f_{i}=a_{i}+b_{i}$, where $a_{i} \in A$ and $b_{i} \in B$. The resulting decomposition of $f$ as element of $R[X]=A[X] \oplus B[X]$ is $f=g+h$, where $g=\sum_{i=0}^{n} a_{i} X^{i}$ and $h=\sum_{i=0}^{n} b_{i} X^{i}$. We prove that $f$ is in the saturation of $\mathscr{S}(R[X])$ by proving that $g$ is in $\mathscr{S}(A[X])$ and $h$ is in the saturation of $\mathscr{S}(B[X])$. Note that $a_{n}=f_{n}$ and $b_{n}=0$ since $f_{n} \in A$; hence $g \in \mathscr{S}(A[X])$ and $h$ has degree less than n. Moreover, $A \oplus B=R=C(f)=C(g) \oplus C(h)$ so that $C(h)=B$ and $h$, considered as an element of the ring $B[X]$, has unit content. To show that $h$ is in the saturation of $\mathscr{S}(B[X])$ it therefore suffices, in view of the induction hypothesis, to prove that $h$ satisfies condition (2) of Proposition 1. Thus, assume that $h$ has degree $m<n$, assume that $j$ is between 0 and $m$, and let $P$ be a prime ideal of the ring $B$ such that the relations $b_{j+1}, \cdots, b_{m} \in P, b_{j} \notin P$ hold. Then $A \oplus P$ is prime in $R$ and the relations $f_{j+1}, \cdots, f_{n} \in A \oplus P, f_{j} \notin A \oplus P$ hold. Since $f$ is a $(*)$-polynomial, it follows that $f_{j}=a_{j}+b_{j}$ is a unit modulo $A \oplus P$, and this implies that $b_{j}$ is a unit modulo $P$. Therefore condition (2) of Proposition 1 is satisfied for the polynomial $h \in B[X]$, and this completes the proof of Theorem 6 .

COROLlaRY 7. The multiplicative system $\mathscr{S}(R[X])$ is saturated if and only if $R$ is indecomposable and reduced.

Proof. Assume first that $R$ is reduced and indecomposable. If $f=\sum_{i=0}^{n} f_{i} X^{i}$ is in the saturation of $\mathscr{S}(R[X])$, where $f_{n} \neq 0$, then 
$f$ is a (*)-polynomial. Since $R$ is reduced, the ideal $\left(f_{n}\right)$ is idempotent. Therefore $\left(f_{n}\right)=R$ since $R$ is indecomposable. It follows that $f_{n}$ is a unit of $R$, and hence $f \in \mathscr{S}(R[X])$. We conclude that $\mathscr{S}(R[X])$ is saturated if $R$ is indecomposable and reduced.

We prove, conversely, that $\mathscr{S}(R[X])$ fails to be saturated if either $R$ is decomposable or $R$ is not reduced. If $R$ is not reduced and if $r$ is a nonzero nilpoint element of $R$, then $1+r X$ is a unit of $R[X]$, hence an element of the saturation of $\mathscr{S}(R[X])$, but $1+r X$ is not in $\mathscr{S}(R[X])$. If $R$ is decomposable and if $1=e_{1}+e_{2}$ is a decomposition of 1 into nonzero orthogonal idempotents, then $e_{1}+e_{2} X$ is in the saturation of $\mathscr{S}(R[X])$ since $\left(e_{1}+e_{2} X\right)\left(e_{2}+e_{1} X\right)=X$, but $e_{1}+e_{2} X$ is not in $\mathscr{S}(R[X])$. This completes the proof of Corollary 7.

COROLlaRY 8. The rings $R\langle X\rangle$ and $R(X)$ coincide if and only if $R$ is zero-dimensional.

Proof. Let $U$ be the multiplicative system of polynomials $f \in R[X]$ of unit content. We note that $R\langle X\rangle=R(X)$ if and only if $U$ is the saturation of $\mathscr{S}(R[X])$. Hence, in view of Theorem 6 , Corollary 8 is equivalent to the statement that each element of $U$ is a (*)-polynomial if and only if $R$ is zero-dimensional. This is the form of Corollary 8 that we choose to establish.

Assume that $R$ is zero-dimensional and that $f \in R[X]$ is a polynomial of unit content. To prove that $f$ is a $(*)$-polynomial, it is sufficient to consider the case where $R$ is reduced. A zero-dimensional reduced ring is von Neumann regular [3, Exer. 12, p. 63], however, so each ideal of $R$ is idempotent. It then follows immediately from the definition that condition (2) of Proposition 1 is satisfied for the polynomial $f$-that is, $f$ is a $(*)$-polynomial. To prove the converse, we show that if $\operatorname{dim} R>0$, then $U$ contains polynomials that are not (*)-polynomials. Thus, let $P$ and $M$ be proper prime ideals with $P \subset M$, and choose $m \in M-P$. Then $f=$ $1+m X \in U$, but $f$ is not a $(*)$-polynomial since, for example, $\phi_{P}^{*}(f)$ is not in the saturation of $\mathscr{S}((R / P)[X])$.

An analysis of the proof of Theorem 6 yields a strong form of the converse of Proposition 5 in the case when $R$ is reduced. The resulting characterization of the elements of the saturation of $\mathscr{S}(R[X])$ is given in the statement of Theorem 9 .

THEOREM 9. Assume that $R$ is a reduced ring, and let $f=f_{0}+$ 
$f_{1} X+\cdots+f_{n} X^{n} \in R[X]$. The following conditions are equivalent.

(1) The polynomial $f$ belongs to the saturation of $\mathscr{S}(R[X])$.

(2) There exists a direct sum decomposition $R=R_{1} \oplus \cdots \oplus R_{m}$ of $R$ such that if $f=g_{1}+\cdots+g_{m}$ is the decomposition of $f$ with respect to the induced decomposition $R[X]=R_{1}[X] \oplus \cdots \oplus R_{m}[X]$ of $R[X]$, then $g_{i} \in \mathscr{S}\left(R_{i}[X]\right)$ for each $i$.

Proof. That (2) implies (1) follows from Proposition 5. To prove the converse, we use induction on $\operatorname{deg} f$. If $\operatorname{deg} f=0$, then $f=f_{0}$ is a unit of $R$ since $f$ has unit content, and hence $f \in \mathscr{S}(R[X])$. We assume that the desired conclusion holds for $\operatorname{deg} f<k$ and we consider the case where $f$ has degree $k$. By Proposition 4, the ideal $\left(f_{k}\right)$ is idempotent. Let $A=\left(f_{k}\right)$, let $B=\operatorname{Ann}\left(f_{k}\right)$, and for each $i$ between 0 and $k$, let $f_{i}=a_{i}+b_{i}$ be the decomposition of $f_{i}$ with respect to the decomposition $R=A \oplus B$ of $R$. Note that $a_{k}=f_{k}$ and $b_{k}=0$ since $f_{k} \in A$, and $f_{k}$ is a unit of the ring $A$ since $f_{k}$ generates the unit ideal of $A$. Let $g=\sum_{i=0}^{k} a_{i} X^{i} \in A[X]$ and let $h=\sum_{i=0}^{k} b_{i} X^{i} \in B[X]$. The observations just made show that $g \in$ $\mathscr{S}(A[X])$ and $\operatorname{deg} h<k$. As $h$ belongs to the saturation of $\mathscr{S}(B[X])$ by Proposition 5, and since $B$ is a reduced ring, the induction hypothesis implies that there exists a direct sum decomposition $B=B_{1} \oplus \cdots \oplus B_{v}$ of $B$ such that if $h=h_{1}+\cdots+h_{v}$ is the decomposition of with respect to the induced decomposition of $B[X]$, then $h_{i} \in \mathscr{S}\left(B_{i}[X]\right)$ for each $i$. By considering the decomposition $R=$ $A \oplus B_{1} \oplus \cdots \oplus B_{v}$ of $R$, we obtain condition (2) for the polynomial $f$.

The analogue of the ring $R(X)$ for polynomials in more than one variable is well known. In fact, the set $U$ of polynomials $f \epsilon$ $R\left[\left\{X_{\lambda}\right\}_{\lambda \in}\right]$ of unit content is a saturated regular multiplicative system in $R\left[\left\{X_{\lambda}\right\}\right]$, and the quotient ring $R\left[\left\{X_{\lambda}\right\}\right]_{U}$ is denoted by $R\left(\left\{X_{\lambda}\right\}\right)$. It would be interesting to have an analogue of the ring $R\langle X\rangle$ for polynomial rings in several variables; for the purpose of this discussion we restrict ourselves to a polynomial ring $R\left[X_{1}, \cdots, X_{n}\right]$ in finitely many variables. Since

$$
R\left(X_{1}, \cdots, X_{i+1}\right)=R\left(X_{1}, \cdots, X_{i}\right)\left(X_{i+1}\right),
$$

where $R\left(X_{1}, \cdots, X_{i}\right)\left(X_{i+1}\right)$ denotes the quotient ring of the polynomial ring $R\left(X_{1}, \cdots, X_{i}\right)\left[X_{i+1}\right]$ with respect to the multiplicative system of polynomials in $X_{i+1}$ of unit content, a natural definition for $R\left\langle X_{1}, \cdots, X_{n}\right\rangle$ is $R\left\langle X_{1}, \cdots, X_{n-1}\right\rangle\left\langle X_{n}\right\rangle$. We note at once that order of the indeterminates is, in general, pertinent in the definition of $R\left\langle X_{1}, \cdots, X_{n}\right\rangle$, while this is not the case for $R\left(X_{1}, \cdots, X_{n}\right)$. That is, 


$$
R\left(X_{1}, \cdots, X_{n}\right)=R\left(X_{\sigma(1)}, \cdots, X_{\sigma(n)}\right)
$$

for each permutation $\sigma$ of $\{1,2, \cdots, n\}$, but $R\left\langle X_{1}\right\rangle\left\langle X_{2}\right\rangle$ need not equal $R\left\langle X_{2}\right\rangle\left\langle X_{1}\right\rangle$, for example, for an arbitrary ring $R$. The next result gives precise information concerning this question of order of the indeterminates.

Proposition 10. Assume that $\left\{X_{i}\right\}_{i=1}^{n}$ is a set of indeterminates over $R$, where $n>1$, and let $\tau$ and $\sigma$ denote distinct permutations of $\{1,2, \cdots, n\}$.

(1) If $R$ is zero-dimensional, then

$$
R\left\langle X_{\tau(1)}, \cdots, X_{\tau(n)}\right\rangle=R\left(X_{1}, \cdots, X_{n}\right)=R\left\langle X_{\sigma(1)}, \cdots, X_{\sigma(n)}\right\rangle .
$$

(2) If $\operatorname{dim} R>0$, then

$$
R\left\langle X_{\tau(1)}, \cdots, X_{\tau(n)}\right\rangle \neq R\left\langle X_{\sigma(1)}, \cdots, X_{\sigma(n)}\right\rangle .
$$

Proof. There is no loss of generality in assuming that $\tau$ is the identity mapping on $\{1,2, \cdots, n\}$. To prove (1), we first prove that for $R$ zero-dimensional, the ring $R\left(X_{1}, \cdots, X_{n}\right)$ is also zero-dimensional (see [1, Lemma 1]). Since

$$
R\left(X_{1}, \cdots, X_{n}\right)=R\left(X_{1}, \cdots, X_{n-1}\right)\left(X_{n}\right),
$$

it suffices to consider the case where $n=1$. Let $P$ be a proper prime ideal of $R[X]$. Since $R$ is zero-dimensional, the ideal $M=P \cap R$ is maximal in $R$. Moreover, it is well known that either $P=M[X]$ or $P=(M[X], f)$ for some monic polynomial $f \in R[X]$ that is irreducible modulo $M$. It follows that the set of proper primes of $R(X)$ is $\left\{M_{\lambda}[X] R(X)\right\}$, where $\left\{M_{\lambda}\right\}$ is the set of maximal ideals of $R$. Hence $\operatorname{dim} R(X)=0$ if $\operatorname{dim} R=0$.

We proceed to establish :(1) by induction on $n$. For $n=1$, Corollary 8 shows that $R\left\langle X_{1}\right\rangle=R\left(X_{1}\right)$. If we assume (1) for $n=k$, then

$$
\begin{aligned}
& R\left\langle X_{\sigma(1)}, \cdots, X_{o(k+1)}\right\rangle=R\left\langle X_{o(1)}, \cdots, X_{\sigma(k)}\right\rangle\left\langle X_{o(k+1)}\right\rangle \\
& \quad=R\left(X_{o(1)}, \cdots, X_{o(k)}\right)\left\langle X_{o(k+1)}\right\rangle .
\end{aligned}
$$

Since $R\left(X_{\sigma(1)}, \cdots, X_{\sigma(k)}\right)$ is zero-dimensional, it follows that

$$
\begin{aligned}
& R\left(X_{\sigma(1)}, \cdots, X_{\sigma(k)}\right)\left\langle X_{\sigma(k+1)}\right\rangle \\
& \quad=R\left(X_{\sigma(1)}, \cdots, X_{\sigma(k)}, X_{\sigma(k+1)}\right)=R\left(X_{1}, \cdots, X_{k+1}\right) .
\end{aligned}
$$

Therefore

$$
R\left\langle X_{\sigma(1)}, \cdots, X_{\sigma(n)}\right\rangle=R\left(X_{1}, \cdots, X_{n}\right)
$$

if $R$ is zero-dimensional. 
To prove (2), we choose $i, j$ such that $i<j$, while $\sigma^{-1}(i)>\sigma^{-1}(j)$. Let $M$ and $P$ be proper prime ideals of $R$ such that $P \subset M$, and choose $m \in M-P$. To prove that $R\left\langle X_{1}, \cdots, X_{n}\right\rangle$ and $R\left\langle X_{\sigma(1)}, \cdots, X_{\sigma(n)}\right\rangle$ are distinct, we show that $f=X_{i}+m X_{j}$ is a unit of the second ring, but not of the first. Since $\sigma^{-1}(j)<\sigma^{-1}(i)$, the integer $j$ precedes $i$ in the listing $\sigma(1), \sigma(2), \cdots, \sigma(n)$. Hence $f$, as an element of

$$
R\left\langle X_{\sigma(1)}, \cdots, X_{o\left(\sigma^{-1}(i)-1\right)}\right\rangle\left[X_{\sigma\left(\sigma^{-1}(i)\right)}\right],
$$

is monic in $X_{i}$. Therefore $f$ is a unit of $R\left\langle X_{\sigma(1)}, \cdots, X_{\sigma(n)}\right\rangle$, as asserted. We prove that $f$ in not a unit of $R\left\langle X_{1}, \cdots, X_{n}\right\rangle$. Proposition 1 shows that the only elements of $R$ that are units of $R\langle X\rangle$ are the units of $R$. Hence, it suffices to show that $f$ is not a unit of

$$
R\left\langle X_{1}, \cdots, X_{j}\right\rangle=R\left\langle X_{1}, \cdots, X_{j-1}\right\rangle\left\langle X_{j}\right\rangle
$$

If $f$ were a unit of $R\left\langle X_{1}, \cdots, X_{j-1}\right\rangle\left\langle X_{j}\right\rangle$, then it follows from Propositions 1-4 that $m$ generates an idempotent ideal of $R\left\langle X_{1}, \cdots\right.$, $\left.X_{j-1}\right\rangle$ modulo its nilradical. Since $P R\left\langle X_{1}, \cdots, X_{j-1}\right\rangle$ is prime in $R\left\langle X_{1}, \cdots, X_{j-1}\right\rangle$, it follows that $m$ generates an idempotent ideal modulo $P R\left\langle X_{1}, \cdots, X_{j-1}\right\rangle$. Hence either $m \in P R\left\langle X_{1}, \cdots, X_{j-1}\right\rangle \cap R=P$ or $m$ is a unit modulo $P R\left\langle X_{1}, \cdots, X_{j-1}\right\rangle$. By choice of $m$, we have $m \notin P$, and $m$ is not a unit modulo $P\left\langle X_{1}, \cdots, X_{j-1}\right\rangle$ since $m$ belongs to the proper ideal $M\left\langle X_{1}, \cdots, X_{j-1}\right\rangle$ of $R\left\langle X_{1}, \cdots, X_{j-1}\right\rangle$. Consequently, $f$ if not a unit of $R\left\langle X_{1}, \cdots, X_{j-1}\right\rangle\left\langle X_{j}\right\rangle$, and this completes the proof of Proposition 10.

Although the order in which the indeterminates are taken in forming $R\left\langle X_{1}, \cdots, X_{n}\right\rangle$ is significant in general, the ring $R\left\langle X_{1}, \cdots, X_{n}\right\rangle$ nevertheless provides a natural analogue of the ring $R\left\langle X_{1}\right\rangle$. In fact, let $Z_{0}$ denote the additive semigroup of nonnegative integers, and let $E_{n}=Z_{0} \oplus \cdots \oplus Z_{0}$ be the direct sum of $n$ copies of $Z_{0}$. The semigroup $E_{n}$ is totally ordered under its reverse lexicographic order-that is, $\left(a_{1}, \cdots, a_{n}\right)<\left(b_{1}, \cdots, b_{n}\right)$ if $a_{i}<b_{i}$ for the last coordinate in which the two elements differ. The polynomial ring $R\left[X_{1}, \cdots, X_{n}\right]$ is isomorphic to the semigroup $\operatorname{ring} R\left[X ; E_{n}\right]$ of $E_{n}$ over $R$ under the isomorphism $\Phi$ that sends $r X_{1}^{e_{1}} \cdots X_{n}^{e_{n}}$ to $r X^{\left(e_{1}, \cdots, e_{n}\right)}$ for each $r \in R$ and for all $e_{1}, \cdots, e_{n} \in Z_{0}$. The elements of $R\left[X ; E_{n}\right]$ can be expressed in the form $r_{1} X^{t_{1}}+\cdots+r_{m} X^{t_{m}}$, where $r_{i} \in R$ for each $i$ and $t_{1}<t_{2}<\cdots<t_{m}$. Hence, the concepts of "leading coefficient" and "monic" are meaningful in $R\left[X ; E_{n}\right]$. Moreover, if $S$ is the set of monic elements of $R\left[X ; E_{n}\right]$, then $S$ is a regular multiplicative system. The results previously proved for the saturation of $\mathscr{S}(R[X])$ carry over to the saturation of $S$; in particular, this is true of Theorems 6 and 9 . For these results, 
the significant property of $Z_{0}$, in considering $R[X]$ as the semigroup ring of $Z_{0}$ over $R$, is that there exists no infinite strictly decreasing sequence $u_{1}>u_{2}>\cdots$ of elements of $Z_{0}$; the semigroups $E_{n}$ share this property, and hence proofs of the theorems extend. The set $\Phi^{-1}(S)$ is the regular multiplicative system in $R\left[X_{1}, \cdots, X_{n}\right]$ corresponding to the set of monic elements of $R\left[X ; E_{n}\right]$, and it is not difficult to verify that $R\left[X_{1}, \cdots, X_{n}\right]_{\Phi^{-1}(S)}=R\left\langle X_{1}, \cdots, X_{n}\right\rangle$. The elements of $\Phi^{-1}(S)$ can be described recursively as follows: the element of $R\left[X_{1}\right]$ in $\Phi^{-1}(S)$ are monic polynonials; given $f \in R\left[X_{1}, \cdots, X_{n}\right]$, we write $f$ as a polynomial in $X_{n}$ with coefficients in $R\left[X_{1}, \cdots, X_{n-1}\right]$, and $f \in \Phi^{-1}(S)$ if and only if the leading coefficient of $f$ is in $\Phi^{-1}(S)$.

In order to clarify the statement of the analogues of Theorems 6 and 9 for semigroup rings, we provide an explicit statement of the extension of these two results.

THEOREM 11. Assume that $E$ is a totally ordered abelian semigroup with zero, and with the property that there exists no infinite strictly decreasing sequence of elements of $E$. Let $S$ be the multiplicative system in $R[X ; E]$ consisting of monic elements, and denote by $S^{*}$ the saturation of $S$. For an element

$$
f=f_{1} X^{e_{1}}+\cdots+f_{n} X^{e_{n}} \in R[X ; E],
$$

where $e_{1}<e_{2}<\cdots<e_{n}$, the following conditions are equivalent.

(1) $f \in S^{*}$.

(2) The coefficients of fgenerate the unit ideal of $R$, and for each $j$ between 1 and $n$ and each prime ideal $P$ of $R$ the relations $f_{j+1}, \cdots, f_{n} \in P, f_{j} \notin P$ imply that $f_{j}$ is a unit modulo $P$.

Moreover, if $R$ is reduced, then (1) and (2) are equivalent to (3).

(3) There exists a direct sum decomposition $R=R_{1} \oplus \cdots \oplus R_{k}$ of $R$ such that if $f=g_{1}+\cdots+g_{k}$ is the decomposition of $f$ with respect to the induced decomposition

$$
R[X ; E]=R_{1}[X ; E] \oplus \cdots \oplus R_{k}[X ; E],
$$

then the leading coefficient of $g_{i}$ is a unit of $R_{i}$ for each $i$ between 1 and $k$.

\section{REFERENCES}

1. J. W. Brewer and D. L. Costa, Projective modules over some non-Noetherian polynomial rings, preprint.

2. Robert Gilmer, Multiplicative Ideal Theory (Marcel Dekker, New York, 1972).

3. Irving Kaplansky, Commutative Rings (Allyn and Bacon, Boston, 1970).

4. Ryuki Matsuda, Infinite group rings III, Bull. Fac. Sci. Ibaraki Univ. Ser. A. Math.,

8 (1976), 1-46. 
5. Masayoshi Nagata, Local Rings (John Wiley, Interscience, New York, 1962).

6. Daniel Quillen, Projective modules over polynomial rings, Inventiones Math., 36 (1976), 167-171.

Received December 8, 1977, and in revised form February 22, 1978. Research partially supported by grants from the National Science Foundation.

Florida State University

Tallahassee, FL 32306

AND

PURDUE UnIVERStTY

W. LAFAYETTE, IN 47907 



\section{PACIFIC JOURNAL OF MATHEMATICS}

\section{EDITORS}

RICHARD ARENS (Managing Editor)

University of California

Los Angeles, California 90024

C. W. CurTis

University of Oregon

Eugene, OR 97403

C. C. MOORE

University of California

Berkeley, CA 94720
J. DUGUNDJI

Department of Mathematics University of Southern California Los Angeles, California 90007

R. FinN AND J. Milgram Stanford University Stanford, California 94305

\section{ASSOCIATE EDITORS}

E. F. BECKENBACH

B. H. NeumanN

F. WOLF

K. YoSHIDA

\section{SUPPORTING INSTITUTIONS}

UNIVERSITY OF BRITISH COLUMBIA CALIFORNIA INSTITUTE OF TECHNOLOGY UNIVERSITY OF CALIFORNIA MONTANA STATE UNIVERSITY UNIVERSITY OF NEVADA, RENO NEW MEXICO STATE UNIVERSITY OREGON STATE UNIVERSITY UNIVERSITY OF OREGON
UNIVERSITY OF SOUTHERN CALIFORNIA

STANFORD UNIVERSITY

UNIVERSITY OF HAWAII

UNIVERSITY OF TOKYO

UNIVERSITY OF UTAH

WASHINGTON STATE UNIVERSITY

UNIVERSITY OF WASHINGTON 


\section{Pacific Journal of Mathematics \\ Vol. 78, No. $1 \quad$ March, 1978}

Simeon M. Berman, A class of isotropic distributions in $\mathbf{R}^{n}$ and their

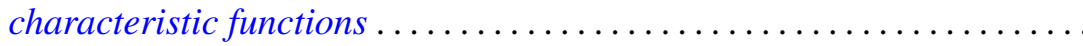

Ezra Brown and Charles John Parry, The 2-class group of biquadratic fields.

II ........................................ 11

Thomas E. Cecil and Patrick J. Ryan, Focal sets of submanifolds ....... 27

Joseph A. Cima and James Warren Roberts, Denting points in $B^{p} \ldots \ldots \ldots 41$

Thomas W. Cusick, Integer multiples of periodic continued fractions . . . . . 47

Robert D. Davis, The factors of the ramification sequence of a class of

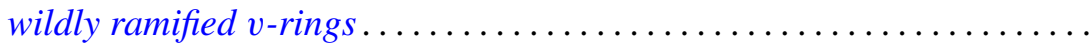

Robert Martin Ephraim, Multiplicative linear functionals of Stein

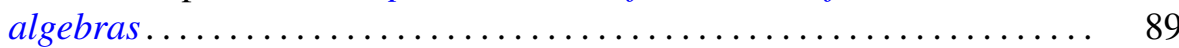

Philip Joel Feinsilver, Operator calculus . .................... 95

David Andrew Gay and William Yslas Vélez, On the degree of the splitting field of an irreducible binomial ..........................

Robert William Gilmer, Jr. and William James Heinzer, On the divisors of

monic polynomials over a commutative ring ..................

Robert E. Hartwig, Schur's theorem and the Drazin inverse .............

Hugh M. Hilden, Embeddings and branched covering spaces for three and four dimensional manifolds ............................

Carlos Moreno, The Petersson inner product and the residue of an Euler product. ...

Christopher Lloyd Morgan, On relations for representations of finite groups....

Ira J. Papick, Finite type extensions and coherence

$\mathrm{R}$. Michael Range, The Carathéodory metric and holomorphic maps on a class of weakly pseudoconvex domains ................

Donald Michael Redmond, Mean value theorems for a class of Dirichlet series

Daniel Reich, Partitioning integers using a finitely generated semigroup ...

Georg Johann Rieger, Remark on a paper of Stux concerning squarefree

numbers in non-linear sequences

Gerhard Rosenberger, Alternierende Produkte in freien Gruppen ..

Ryōtarō Satō, Contraction semigroups in Lebesgue space

Tord Sjödin, Capacities of compact sets in linear subspaces of $\mathbf{R}^{n}$

Robert Jeffrey Zimmer, Uniform subgroups and ergodic actions of exponential Lie groups......................... 